\title{
A LOW-MACH/ACOUSTIC SOLVER FOR FLUID STRUCTURE INTERACTION PROBLEMS
}

\author{
Cong-Huan PHAN ${ }^{1,2}$, Alberto BECCANTINI ${ }^{1}$ AND Christophe CORRE ${ }^{2}$ \\ ${ }^{1}$ Université Paris-Saclay, CEA, Service d'études mécaniques et thermiques \\ 91191 Gif-sur-Yvette, France \\ cong-huan.phan@cea.fr, alberto.beccantini@cea.fr \\ ${ }^{2}$ Ecole Centrale de Lyon, LMFA UMR 5509 \\ 69134 Ecully, France \\ cong-huan.phan@ec-lyon.fr, christophe.corre@ec-lyon.fr
}

Key words: low-Mach regime, all-Mach correction, stiffened gas, Gresho vortex, shock tube, piston problem

\begin{abstract}
An all-Mach correction is applied to the HLLC scheme for computing liquid flows described by the Euler equations closed with the stiffened gas EoS. The accuracy provided by the corrected scheme is assessed on a series of model problems : Gresho vortex, low-Mach shock tube and a piston problem providing a simplified description of the bubble expansion occurring in some nuclear safety problems.
\end{abstract}

\section{INTRODUCTION}

The scenario of an Hypothetical Core Disruptive Accident (HCDA) for a liquid-sodium fast breeder reactor supposes the reactor core has partially melted and the molten fuel chemically interacts with the liquid sodium to produce a large quantity of gaseous components [1]. The explosive expansion of the high-pressure gas bubble formed in the core centre yields significant loads on the reactor vessel, which have been studied through a number of experimental programs. The MARA set of experiments was carried out by CEA in the 1980s on a $1 / 30$-scale model of the Superphenix reactor in order to provide reference measurements (pressure at transducers, structure deformation) to be used for validation of the codes applied to the HCDA fluid-structure interaction problem. In the experiments, water replaces the liquid sodium cooling the reactor core and the cover gas below the reactor roof is air instead of argon; the explosion is triggered by an explosive charge (see Fig.1).

Two speed scales are associated with the flow field evolution: the sound speed $c$ and the flow speed $u=M c$ with a local Mach number $M$ which can be much lower than unity in some regions of the computational domain. In the first stage of the bubble expansion, shock waves propagate in the liquid phase and induce a low-Mach motion of the liquid. In a second stage the impact of the liquid on the containment yields structural loads which must be accurately computed. The present work is focused on numerical issues which arises when computing an HCDA-type bubble expansion problem with standard compressible CFD codes based on conservative finite volume methods and Godunov-type numerical fluxes. Such fluxes are robust enough to compute shock waves but become inaccurate for the computation of low-Mach number flows. The predicted kinetic energy can be subjected to an excessive numerical 


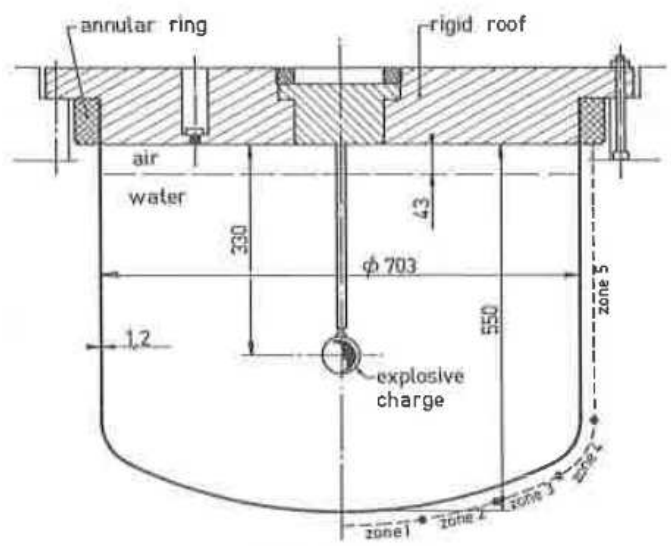

Figure 1: Schematic view of the MARA 1/2 configuration taken from [2]. The charge creating the gas bubble is located at the center of the liquid domain; the layer of air below the vessel cover is also visible.

dissipation, leading to a possible underestimation of the liquid impact on the structure. This accuracy issue can be fixed by a centering of the pressure instead of upwinding in the numerical discretization. The all-Mach number correction proposed in [3] for Godunov-type schemes is adapted in the present work to deal with the stiffened gas model used to describe the liquid phase. Its performance is assessed on models problems representative of the targeted HCDA application. Section 2 reviews the key features of the numerical solver - behavior of a standard upw develop a corrected sch standard and corrected in section 4 .

\section{LOW-MACH/ACOUSTIC SOLVER}
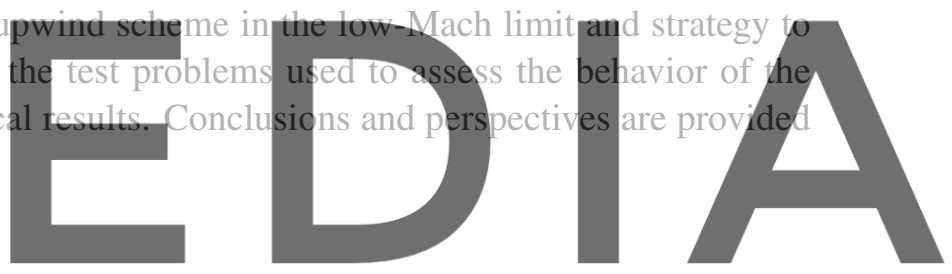

2.1 Behavior in the low Mach number limit

Register for free at https//www.scipedia.com to download the version without the watermark The compressible Euler equations closed by the stiffened gas Equation of State are solved to describe the liquid phase. When applying an asymptotic analysis to analyze the flow problem in the low Mach limit, the pressure behavior for the Euler system at the continuous level is known to be such that :

$$
p=P(t)+\underbrace{p^{\prime}(x, t)}_{o\left(M^{2}\right)}, \quad p^{\prime}(x, t)<<P(t)
$$

At the discrete level, it has been established [4] that for standard upwind schemes applied in the low Mach limit :

$$
p=P(t)+\underbrace{p^{\prime}(x, t)}_{o(M)}, \quad p^{\prime}(x, t) \approx \rho c \Delta u
$$

which means the computed flow contains parasite waves of acoustic nature, wrongly scaled at $o(M)$ instead of $o\left(M^{2}\right)$.

The numerical flux for any standard $X$ upwind scheme ( $X=$ HLLC, Godunov $\ldots$ ) can be recast in the 
form:

$$
F_{i j}^{X}=\frac{1}{2}\left(F_{i}+F_{j}\right)-\underbrace{\frac{1}{2} D^{X} \cdot\left(U_{j}-U_{i}\right)}_{\text {numerical viscosity }}, D^{X} \text { : upwinding matrix }
$$

If $\left(^{*}\right)$ indicates the solution of the (exact or approximate) Riemann problem at the cell interface, then :

$$
F_{i j}^{X}=\left(\begin{array}{c}
\rho^{*}\left(\mathbf{v}^{*} \cdot \mathbf{n}\right) \\
\rho^{*}\left(\mathbf{v}^{*} \cdot \mathbf{n}\right) \mathbf{v}^{*}+p^{*} \mathbf{n} \\
\left(\rho^{*} e^{* t}+p^{*}\right)\left(\mathbf{v}^{*} \cdot \mathbf{n}\right)
\end{array}\right)_{i j}
$$

In the low-Mach regime, the pressure solution of the standard $\mathrm{X}$ scheme reduces to:

$$
p_{i j}^{*}=\frac{1}{2}\left(p_{i}+p_{j}\right)-\underbrace{\frac{\rho c}{2}\left(u_{j}-u_{i}\right)}_{\text {numerical viscosity }}
$$

It is thus clearly observed a correction must be applied to the numerical flux so as to rescale the parasite waves to $O\left(M^{2}\right)$.

\subsection{Numerical flux and all-Mach correction}

Dellacherie et al. have proposed in [3] an all-Mach correction which reads :
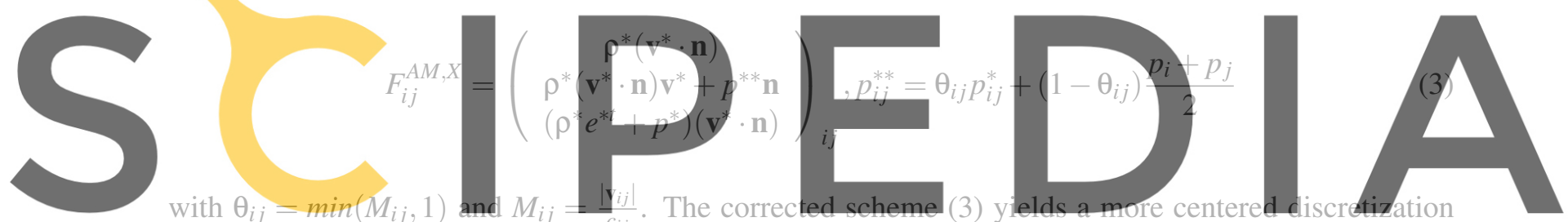

(with a reduced numerical viscosity) than the standard scheme in the low-Mach limit. In the supersonic

Register fogifne,e日; this correction is applied to the HLLC scheme for the Euler equations closed with the stiffened gas EoS to describe the liquid flow. Second-order accuracy is achieved through a MUSCL reconstruction (including Barth-Jespersen slope limiter).

\section{TEST PROBLEMS}

\subsection{Overview}

The standard and corrected HLLC schemes described in the previous section are now applied to solve the Euler equations for liquid (water) flows, with water described by the stiffened gas equation of state $\left(\gamma=4.4, p_{\infty}=6 \times 10^{8} \mathrm{~Pa}\right)$ and initial and boundary conditions successively defining the 3 following test problems: i) a low-Mach number vortex problem is first computed on quadrangular and triangular grids in order to also assess the influence of grid topology [5] on the schemes' ability to compute lowspeed liquid motion; ii) a water shock tube is computed next, to assess the schemes' ability to describe wave propagation; iii) the schemes are eventually inserted in an ALE framework to compute a 2D piston problem representative of the HCDA bubble expansion. 


\subsection{Gresho vortex problem}

The initial condition of the Gresho vortex problem is illustrated in Fig.2. The Gresho vortex is a slowly rotating vortex corresponding to a steady solution of the incompressible Euler equations. The vortex should be consequently preserved over time and the deviations observed in the evolution from its initial state are directly produced by the discretization error of the applied numerical scheme. The ratio between the rotational velocity and the speed of sound defines the characteristic Mach number of the flow; its maximum value is $M_{\max }=5.0 e-4$. Note this problem has been previously used by other authors [6] [7] [8] to assess the properties of numerical schemes in the low Mach regime for perfect gas simulations. In the present work, the stiffened gas EoS is used to describe the water flow. Typical results obtained on

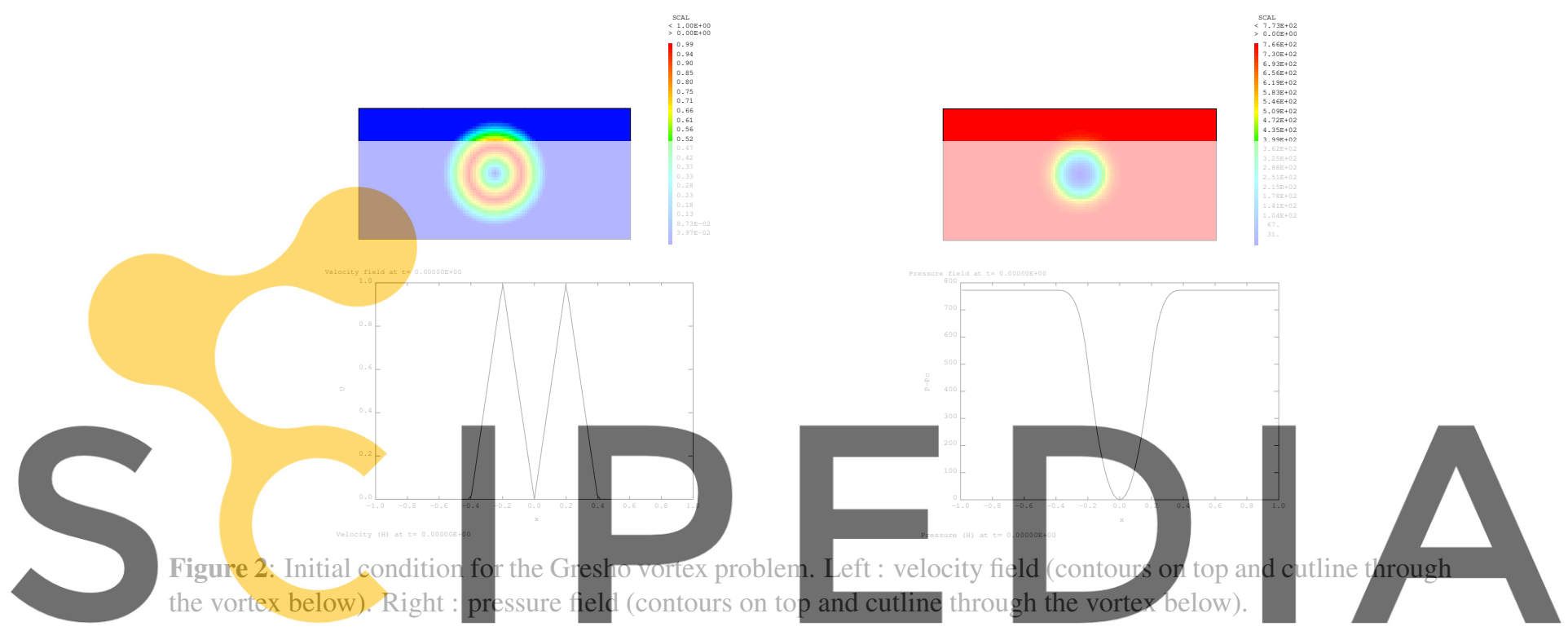

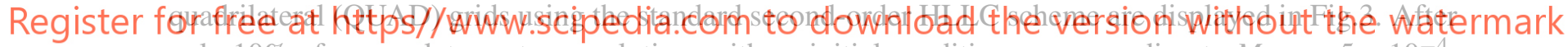 only $10 \%$ of a complete vortex revolution, with an initial condition corresponding to $M_{\max }=5 \times 10^{-4}$,} the expected excessive diffusion of the standard scheme is clearly observed while the corrected scheme yields a properly scaled diffusion in the low-Mach limit and well preserves the initial condition.

Computations are also performed on triangular (TRI) grids and it is observed the observations made by [5] are also well recovered for the present stiffened gas calculation : TRI grids allow to prevent excessive numerical diffusion of velocity for the standard scheme but do not fully correct the occurrence of pressure oscillations (see Table 1). In the perspective of HCDA calculation, the proper conservation of the liquid kinetic energy must be ensured so as to preserve the correct assessment of the structural loads. Fig.4 displays the time evolution of kinetic energy for the Gresho vortex when the standard scheme is applied on a QUAD or a TRI grid and when the corrected scheme is applied on a QUAD grid. Ideally, the kinetic energy normalized by its initial level should remain equal to unity. It is thus evidenced the corrected scheme does a very good job of preserving the kinetic energy of the vortex. Meanwhile, the standard scheme quickly dissipates this kinetic energy when applied on QUAD grids. Using TRI grids significantly improves the behavior of the standard scheme in the low Mach regime, even though this numerical strategy remains less accurate than the corrected scheme. 


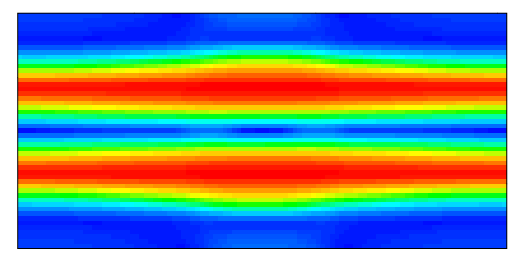

Standard
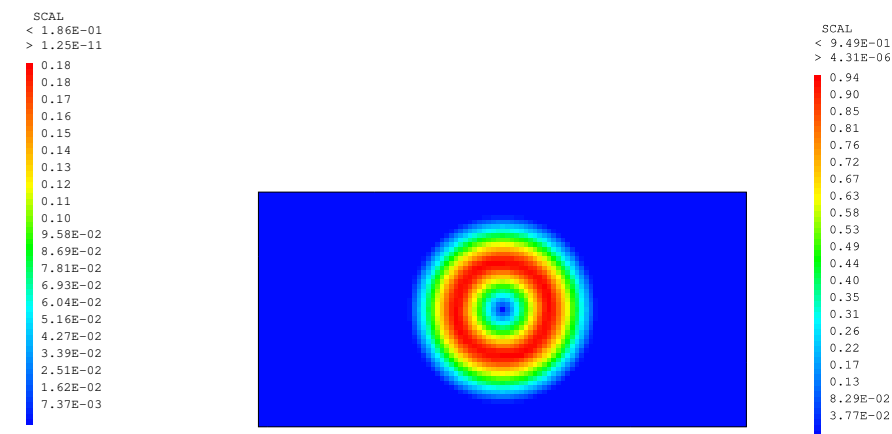

Velocity field at $t=1.25000 \mathrm{E}-0$

Figure 3: Gresho vortex problem. Contours of velocity after $10 \%$ of complete revolution from the initial state $\left(M_{\max }=5 \times 10^{-4}\right)$. QUAD grid calculation. Left : standard scheme. Right : corrected scheme.

\begin{tabular}{|c|c|c|c|c|c|c|}
\hline \hline Scheme & \multicolumn{5}{|c|}{ Water Gresho vortex test case } \\
\hline & \multicolumn{3}{|c|}{ QUAD } & \multicolumn{3}{c|}{ TRI } \\
\hline & Density & Velocity & Pressure & Density & Velocity & Pressure \\
\hline Standard & $\checkmark$ & $\times$ & $\times$ & $\checkmark$ & $\checkmark$ & $\times$ \\
\hline Corrected & $\checkmark$ & $\checkmark$ & $\checkmark$ & $\checkmark$ & $\checkmark$ & $\checkmark$ \\
\hline
\end{tabular}

Table 1: Computation of the Gresho vortex on QUAD and TRI grids using the standard and corrected HLLC scheme. Numerical issues ar
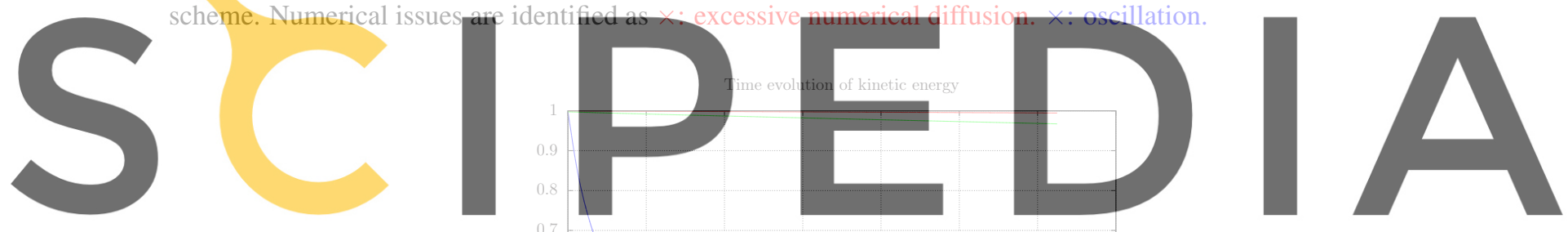

Register for free at https//www.scipedia.com to download the version without the watermark

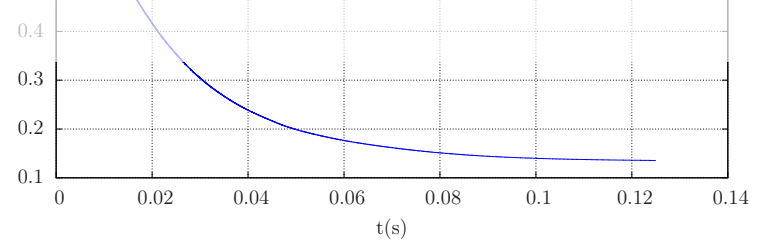

Figure 4: Gresho vortex problem. Evolution of kinetic energy over time.

\subsection{D low-Mach shock tube problem}

The initial right (low pressure) state for water (still described using the stiffened gas EoS) is set as:

$$
\left\{\begin{array}{l}
\rho_{R}^{0}=1000 \mathrm{~kg} / \mathrm{m}^{3} \\
p_{R}^{0}=10^{5} \mathrm{~Pa} \\
u_{R}^{0}=0 \mathrm{~m} / \mathrm{s}
\end{array}\right.
$$


while the initial left (high pressure) state is computed as:

$$
\left\{\begin{array}{l}
\rho_{L}^{0}=\left(1+2 M_{\text {ref }}\right) \times \rho_{R}^{0} \\
p_{L}^{0}=p_{R}^{0}+M_{\text {ref }} \times 2 \rho_{R}^{0}\left(c_{R}^{0}\right)^{2} \\
u_{L}^{0}=u_{R}^{0}
\end{array}\right.
$$

with $c_{0}^{R}=\sqrt{\gamma \frac{p_{0}^{R}+p_{\infty}}{\rho_{R}^{0}}}$. The Mach number $M_{\text {ref }}$ can be varied in order to assess the behaviour of the schemes in the low Mach limit.

The shock tube problem is first computed with $M_{\text {ref }}=10^{-1}$. As expected, no problems are observed with the standard schemes. Fig.5 displays the solution computed using the corrected HLLC scheme at $t_{\text {fin }}=4 \times 10^{-4} \mathrm{~s}$. Oscillations appear near the shock on the QUAD grid; they are damped on the TRI grid. The 1D low-Mach shock tube problem is computed next with $M_{r e f}=10^{-3}$ using the corrected scheme.

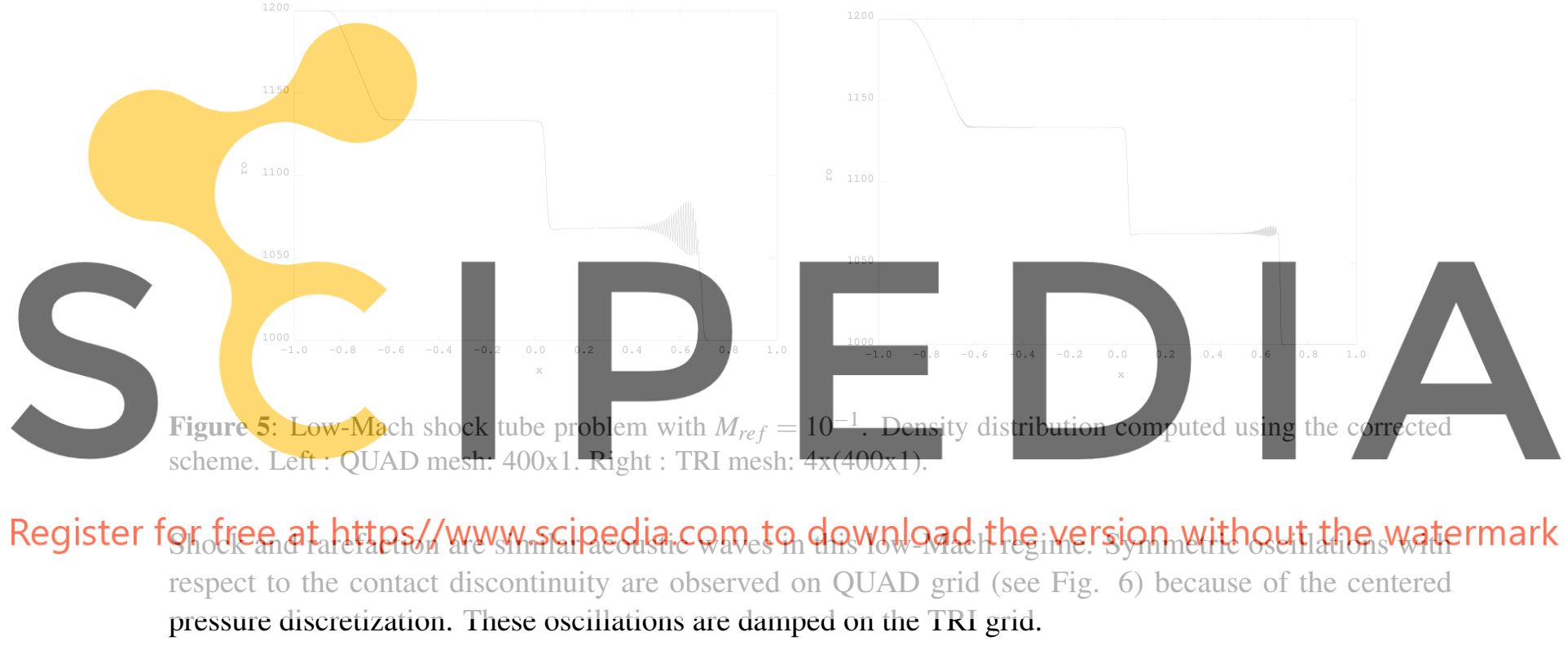

\subsection{D cylindrical axisymmetric piston problem}

The piston problem described in Fig.7 is representative of the bubble expansion characterizing the HCDA application. The piston pushes water, with a characteristic Mach number $M_{p}$ defined as the ratio between the piston velocity and the initial soundspeed. The initial domain is such that $R_{0}=0.1 \mathrm{~m}, R_{\text {ext }}=1 \mathrm{~m}$ and is filled with water (described again using the stiffened gas EoS). A reference solution can be obtained using 1D cylindrical coordinates. The standard and corrected schemes are inserted within an Arbitrary Lagrangian Eulerian (ALE) formulation to deal with the inner moving wall and the 2D numerical solutions obtained for $M_{p}=0.006$ are compared with the reference 1D solution. An unstructured quasiisotropic mesh is used for the 2D computations. Figure 8 displays the flow computed using the standard HLLC scheme, at $t=5 \times 10^{-4}$ s i.e. before the first wave-reflection on the outer (fixed) wall takes place. The $2 \mathrm{D}$ solution provided by the standard scheme compares well with the $1 \mathrm{D}$ reference solution. The flow computed using the standard HLLC, at $t=9.95 \times 10^{-3} s$ i.e. after about 20 reflections on the fixed 

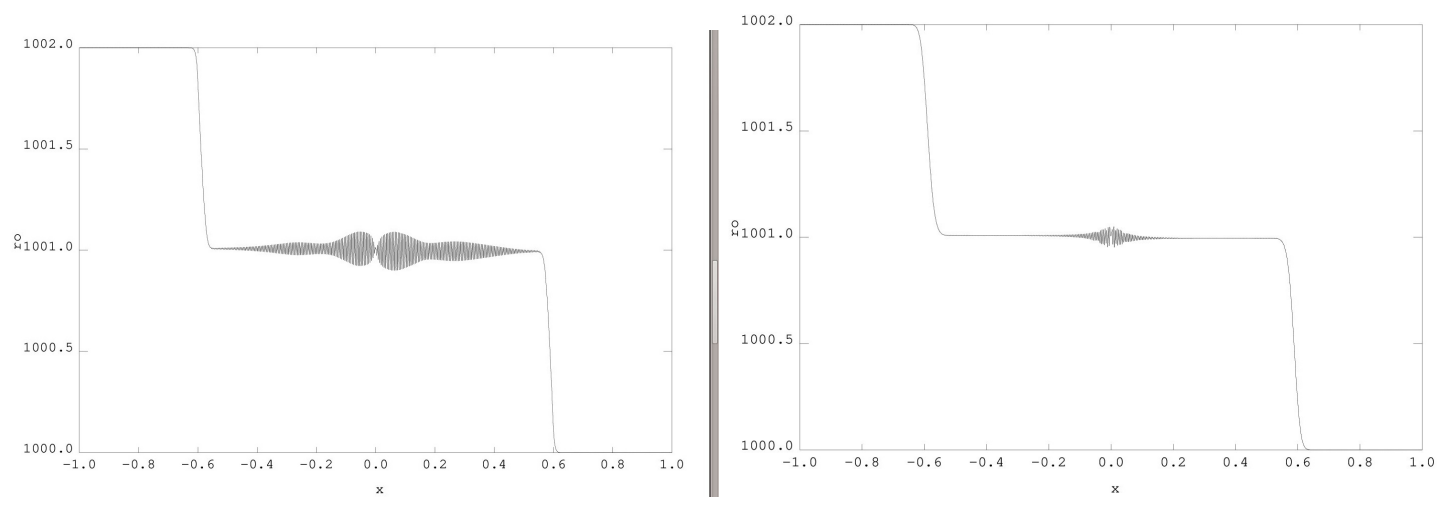

Figure 6: Low-Mach shock tube problem with $M_{r e f}=10^{-3}$. Density distribution computed using the corrected scheme. Left : QUAD mesh: 400x1. Right : TRI mesh: 4x(400x1).
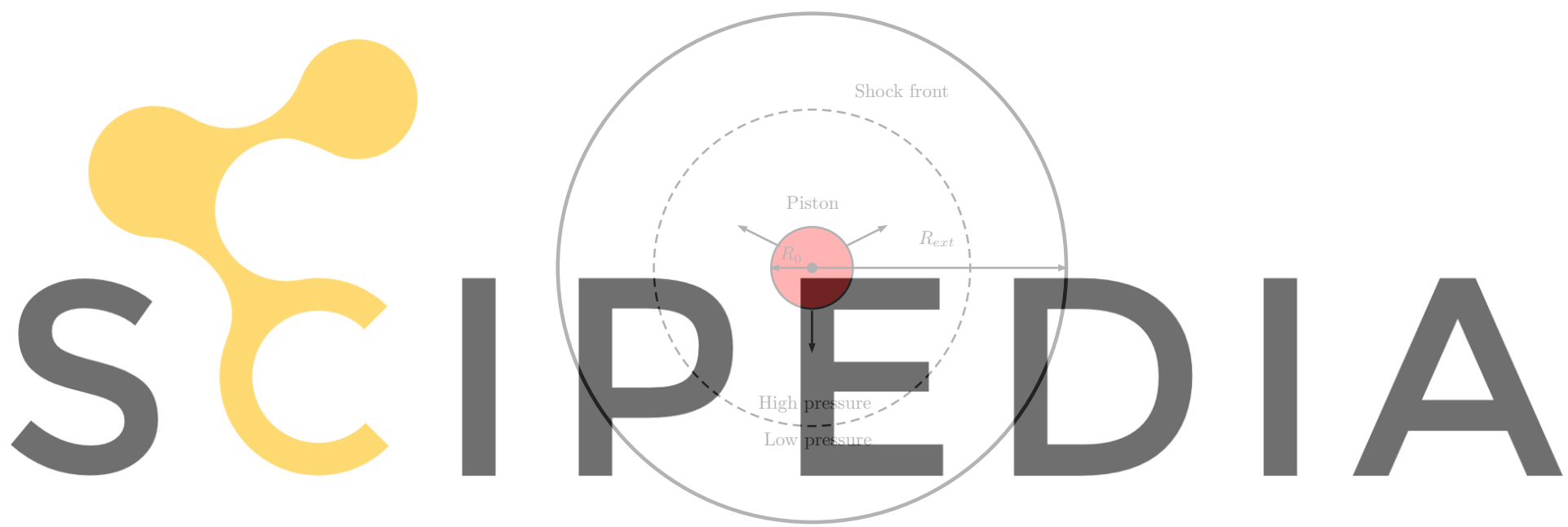

Register for free at htths//www.scipedia.com to download the version without the watermark

outer wall, is displayed in Fig. 9 and compared with the 1D reference solution. The under-estimation of the pressure and velocity peaks, diffused for this long-time observation, is clearly evidenced. For this piston problem, it is therefore observed that no numerical low-Mach problem is appearing. A grid convergence analysis shows that the convergence rate does not deteriorate as the piston Mach number decreases : second-order accuracy of the standard HLLC scheme with MUSCL reconstruction is well preserved. As expected, the acoustic wave is (numerically) dissipated in long time but the parasite waves remain negligible beyond the physical compression wave.

\section{CONCLUSIONS}

The accuracy problem associated with the application of standard compressible solvers making use of approximate Riemann solvers (such as the HLLC scheme) in the low-Mach regime is investigated for low-Mach liquid flows with water described using the stiffened gas EoS. For the Gresho vortex, providing a focus on the velocity scale $u$, it is found an exceedingly dissipated solution is obtained with the standard 

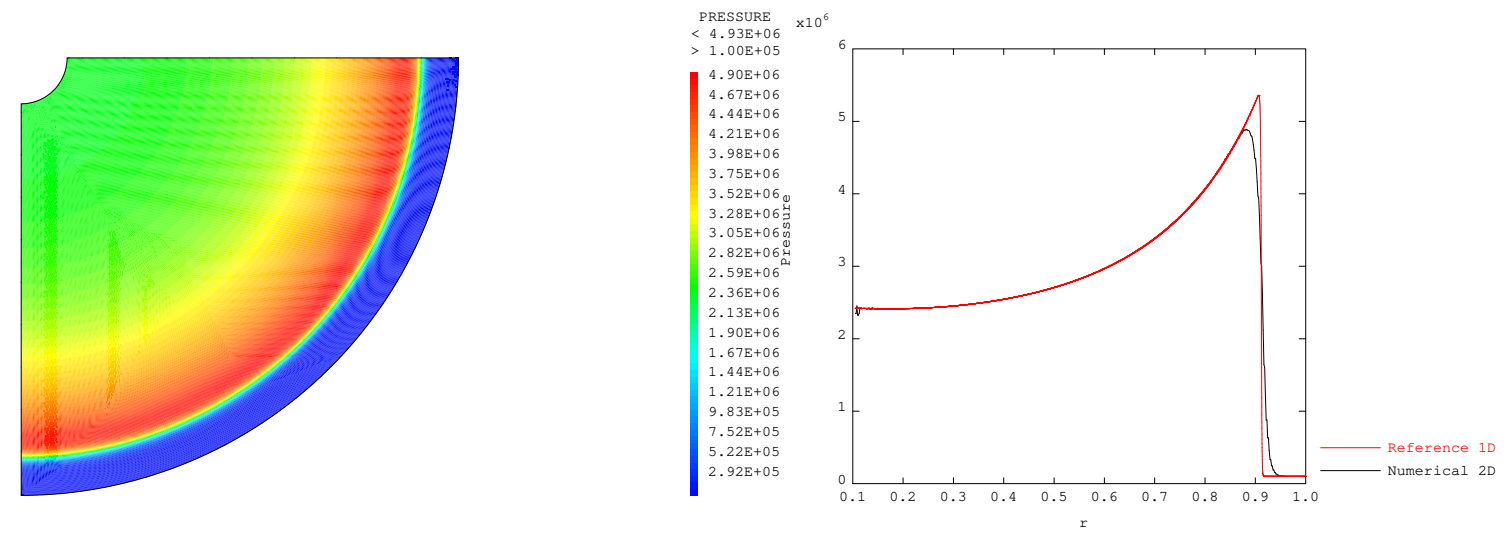

2D pressure at time $5.00000 \mathrm{E}-04$

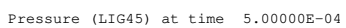

Figure 8: Piston problem $\left(M_{p}=0.006, t=5 \times 10^{-4} \mathrm{~s}\right.$. Standard scheme calculation. Left: pressure contours. Right: pressure distribution (black line) along the line $\theta=-45^{\circ}$.
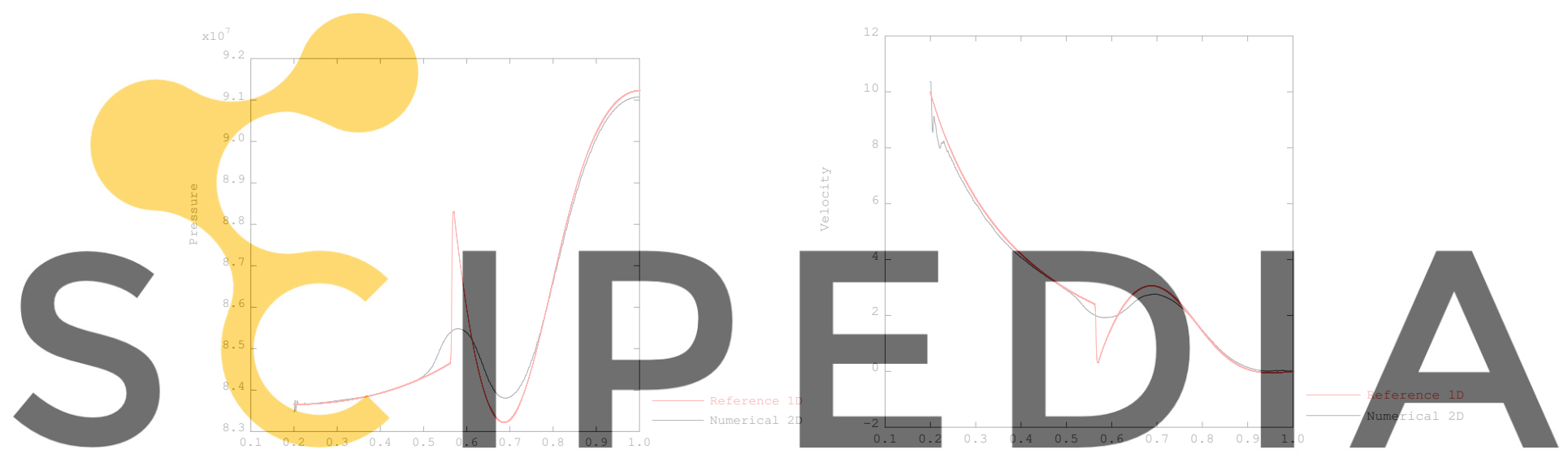

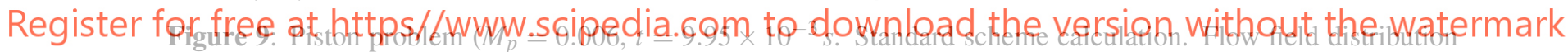
along $\theta=-45^{\circ}$. Left: pressure. Right: velocity

scheme used on a QUAD grid. The underestimated kinetic energy would thus lead to a poor estimation of the loads applied on the containment. Meanwhile, the kinetic energy of the vortex is very well preserved, on the same QUAD grid, when the corrected scheme is applied. For the 1D low-Mach shock tube, providing a focus on the acoustic scale $c$, oscillating solutions are observed when using the corrected schemes on QUAD mesh. However the oscillations are reduced when turning to a TRI mesh. For the piston problem, where the focus is again on the acoustic scale $c$, no accuracy problem is identified with the standard scheme. It can be concluded from these experiments that when both $u$ and $c$ scales coexist in the flow, optimal accuracy is obtained with the low-Mach corrected scheme applied on triangular meshes. If the corrected schemes brings the expected accuracy improvement (on TRI grids), the efficiency of the compressible simulation remains nonetheless poor in the low-Mach regime. Note the correct capture of the acoustic waves is not needed to ensure a conservative estimate of the structural loads for HCDA as those are mostly induced by liquid motion. Consequently, an efficiency improvement is expected from a new physical model based on artificial compressibility and currently under development. 


\section{REFERENCES}

[1] Chang, Y.W.. Analysis of HCDA. Nuclear Engineering and Design 69 (1982) 345-358.

[2] Fiche, C., Louvet, J., Smith, B.L., Zucchini, A., Theoretical experimental study of flexible roof effects in an HCDA's simulation. In: Proceedings of the 8th International Conference on Structural Mechanics in Reactor Technology, Paper E 4/5, Brussels, Belgium, pp. 139-144 (1985).

[3] Dellacherie, S., Jung, J., Omnes, P., and Raviart, P.-A., Construction of modified Godunov-type schemes accurate at any Mach number for the compressible Euler system. Mathematical Models and Methods in Applied Sciences (2016) 26 2525-2615.

[4] Guillard, H., Viozat, C., On the behaviour of upwind schemes in the low Mach number limit. Computers \& Fluids 28 (1999) 63-86.

[5] Rieper, F., Bader, G., The influence of cell geometry on the accuracy of upwind schemes in the low Mach number regime. Journal of Computational Physics (2009) 228 2918-2933.

[6] Miczek, F., Roepke, F., and Edelmann, P. New numerical solver for flows at various Mach numbers. Astronomy \& Astrophysics (2015) $\mathbf{5 7 6 .}$

[7] Barsukow, W., Edelmann, P., Klingenberg, C., Miczek, F. and Röpke, F. A Numerical Scheme for the Compressible Low-Mach Number Regime of Ideal Fluid Dynamics. Journal of Scientific Computing (2017) 72:623-646.

[8] Bruel, P., Delmas, S., Jung, J. and Perrier, V. A low Mach correction able to deal with low Mach
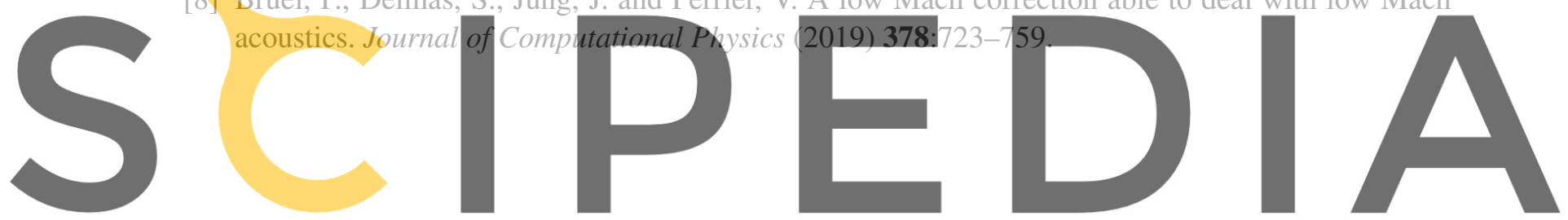

Register for free at https//www.scipedia.com to download the version without the watermark 